\title{
WOULD YOU LIKE TO ENTER FIRST WITH A LOW-QUALITY GOOD?
}

Luca Lambertini ${ }^{\S}$ and Piero Tedeschi ${ }^{\#}$

$\S$ Dipartimento di Scienze Economiche

Università di Bologna

Strada Maggiore 45, 40125 Bologna, Italy

fax: 0039-051-2092664; lamberti@spbo.unibo.it

\# Dipartimento di Statistica

Università di Milano-Bicocca

Dipartimento di Statistica

Via Bicocca degli Arcimboldi, 8, 20126 Milano, Italy fax: 0039-02-6473312; piero.tedeschi@unimib.it

December 3, 2003 


\begin{abstract}
Using a two-period duopoly model with vertical differentiation, we show that there exists a unique subgame perfect equilibrium where the first entrant supplies a lower quality and gains higher profits than the second entrant. We also prove that this entry sequence is also socially efficient.
\end{abstract}

JEL Classification: C73, D43, L13

Keywords: entry, vertical differentiation 


\section{Introduction}

According to the established wisdom concerning vertically differentiated markets, earlier entrants appropriate the high-quality niches, while later entrants fill the remaining lower part of the quality spectrum (Gabszewicz and Thisse, 1979, 1980; Shaked and Sutton, 1982, 1983; Donnenfeld and Weber, 1992; Lehmann, 1997). This is due to two basic assumptions according to which the distribution of cumsumers' willingness to pay is uniform and the game unravels in a single period.

Here, we want to relax the second assumption, by adopting a simple two-period setup, with sequential entry. ${ }^{1}$ Using a model whose original formulation is in Cremer and Thisse (1991), we show that profit incentives drive firms toward a unique subgame perfect equilibrium where the first entrant supplies a lower quality and gains higher profits as compared to the second entrant. Moreover, we also prove that this entry sequence is also socially efficient, in that it entails a higher average quality level than the alternative one. The remainder of the note is structured as follows. The setup is laid out in section 2. The entry process and the welfare performance are investigated in section 3 .

\subsection{The model}

The market exists over two periods, $t \in\{0,1\}$. In each period, a population of consumer of unit size is uniformly distributed over the interval $[\underline{\theta}, \bar{\theta}]$, with $\underline{\theta}=\bar{\theta}-1$. Parameter $\theta \in[\underline{\theta}, \bar{\theta}]$ measures a consumer's marginal willingness

\footnotetext{
${ }^{1}$ In the above mentioned literature, entry is euristically considered in one-shot games, without allowing any explicit role for calendar time. One exception is Dutta et al. (1995) where, however, a high-quality advantage obtains.
} 
to pay for quality, and the net surplus from consumption is:

$$
U=\theta q_{i}-p_{i} \geq 0
$$

where $p_{i}$ and $q_{i}$ are the price and quality of the product supplied by firm $i$. We assume that (1) holds for all consumers in both periods, so that the market is always fully covered.

On the supply side, any firm $i$ must bear total cost $C_{i}=c q_{i}^{2} x_{i}$ per period, where $x_{i}$ is the market demand for her product and $c$ is a positive parameter. Accordingly, firm $i$ 's profit function is $\pi_{i}=\left(p_{i}-c q_{i}^{2}\right) x_{i}$ in each period.

In the remainder, we will consider the following game. Each firm irreversibly sets quality at the time of entry. At $t=0$, the firm 1 enters and remains a monopolist in that period. At $t=1$, firm 2 enters and the market becomes a duopoly. Hence, the problem of the first entrant (the leader) consists in choosing whether to offer a low- or high-quality good, correctly anticipating the optimal behaviour of the second entrant (the follower). That is, the stage describing quality choices is going to be solved à la Stackelberg. Once both qualities are set, Bertrand-Nash competition takes place.

The objective of the leader (firm 1) is:

$$
\max _{p_{M}, p_{1}, q_{1}} \Pi_{1} \equiv \pi_{1}^{M}+\delta \pi_{1}^{D}=p_{M}-c q_{1}^{2}+\delta\left(p_{1}-c q_{1}^{2}\right) x_{1}
$$

where $\pi_{1}^{M}=p_{M}-c q_{1}^{2}$ are monopoly profits at $t=0, \pi_{1}^{D}=\left(p_{1}-c q_{1}^{2}\right) x_{1}$ are duopoly profits at $t=1$, the latter being discounted by the factor $\delta \equiv$ $1 /(1+\rho)$, with $\delta \in[0,1]$ for all $\rho \in[0, \infty)$. The objective of the follower (firm 2) consists in maximising duopoly profits $\pi_{2}^{D}=\left(p_{2}-c q_{2}^{2}\right) x_{2}$ w.r.t. $p_{2}$ and $q_{2}$.

The two firms will supply qualities $q_{H} \geq q_{L}>0$ at duopoly prices $p_{H} \geq$ $p_{L}$, and either $q_{1}=q_{L} ; q_{2}=q_{H}$ or the opposite. In either case, at $t=1$, the 
consumer indexed by $\widehat{\theta}=\left(p_{H}-p_{L}\right) /\left(q_{H}-q_{L}\right)$ will be indifferent between the two goods, so that we may define duopoly demands as follows:

$$
x_{H}=\bar{\theta}-\frac{p_{H}-p_{L}}{q_{H}-q_{L}} ; x_{L}=\frac{p_{H}-p_{L}}{q_{H}-q_{L}}-(\bar{\theta}-1) .
$$

\subsection{Optimal pricing behaviour}

The optimal monopoly pricing at $t=0$ can be quickly characterised, for any given $q_{1}$. Under full coverage, firm 1 sets the price driving to zero the net surplus of the poorest consumer located at $\underline{\theta}=\bar{\theta}-1$, i.e., $p_{M}=(\bar{\theta}-1) q_{1}$. Hence, monopoly profits are $\pi_{1}^{M}=(\bar{\theta}-1) q_{1}-c q_{1}^{2}$.

The Nash game in prices is well known; therefore we omit a detailed exposition (see Cremer and Thisse, 1991, and Lambertini, 1996, inter alia). Equilibrium prices are:

$$
p_{H}=\frac{\left(q_{H}-q_{L}\right)(\bar{\theta}+1)+2 c q_{H}^{2}+c q_{L}^{2}}{3} ; p_{L}=\frac{\left(q_{H}-q_{L}\right)(2-\bar{\theta})+2 c q_{L}^{2}+c q_{H}^{2}}{3}
$$

so that profit functions simplify as follows:

$\pi_{H}=\frac{\left(q_{H}-q_{L}\right)\left[\bar{\theta}+1-c\left(q_{H}+q_{L}\right)\right]^{2}}{9} ; \pi_{L}=\frac{\left(q_{H}-q_{L}\right)\left[2-\bar{\theta}+c\left(q_{H}+q_{L}\right)\right]^{2}}{9}$.

Hence, we have two alternative scenarios. The first, where $q_{1}=q_{L}$ and $q_{2}=q_{H}$, is labelled as low-quality leadership; the second, where $q_{1}=q_{H}$ and $q_{2}=q_{L}$, is labelled as high-quality leadership. 


\section{Entry}

\subsection{Low-quality leadership}

In this case, $\pi_{1}^{D}=\pi_{L}$ and $\pi_{2}^{D}=\pi_{H}$. The leader's problem consists in: ${ }^{2}$

$$
\begin{aligned}
\max _{q_{L}} \Pi_{1 L} & =(\bar{\theta}-1) q_{L}-c q_{L}^{2}+\frac{\left(q_{H}-q_{L}\right)\left[2-\bar{\theta}+c\left(q_{H}+q_{L}\right)\right]^{2}}{9(1+\rho)} \\
\text { s.t. } & : \frac{\partial \pi_{H}}{\partial q_{H}}=0 \Leftrightarrow q_{H}^{*}=\frac{\bar{\theta}+1+c q_{L}}{3 c}
\end{aligned}
$$

Plugging $q_{H}^{*}$ into $\Pi_{1 L}$ and solving the first order condition (FOC) $\partial \Pi_{1 L} / \partial q_{L}=$ 0 w.r.t. $q_{L}$, we obtain:

$$
q_{L}=\frac{16 \bar{\theta}-81 \rho-113 \pm 3 \sqrt{9 \rho(81 \rho+194)+1081}}{32 c}
$$

with

$$
\lim _{\rho \rightarrow \infty} q_{L}^{-}=-\infty ; \lim _{\rho \rightarrow \infty} q_{L}^{+}=\frac{\bar{\theta}-1}{2 c}
$$

the latter being the single-period optimal monopoly quality (see Lambertini, 1997). Therefore, Stackelberg equilibrium qualities are:

$$
\begin{aligned}
q_{L}^{\mathfrak{l}} & =\frac{16 \bar{\theta}-81 \rho-113 \pm 3 \sqrt{9 \rho(81 \rho+194)+1081}}{32 c} \\
q_{H}^{\mathfrak{f}} & =\frac{16 \bar{\theta}-27(1+\rho)+\sqrt{9 \rho(81 \rho+194)+1081}}{32 c}
\end{aligned}
$$

where superscripts $\mathfrak{l}$ and $\mathfrak{f}$ stand for leader and follower, respectively. The associated equilibrium profits are:

$$
\begin{aligned}
\Pi_{1 L}= & \left\{1152 \bar{\theta}(\bar{\theta}-2)(1+\rho)-\rho\left(19683 \rho^{2}+70713 \rho+84969\right)+\right. \\
& \left.-33427+\Psi^{3}\right\} /[4608 c(1+\rho)]
\end{aligned}
$$

\footnotetext{
${ }^{2}$ There exists another solution to $\partial \pi_{H} / \partial q_{H}=0$, i.e., $q_{H}=\left(\bar{\theta}+1-c q_{L}\right) / c$. However, this can be excluded on the basis of concavity conditions.
} 


$$
\pi_{H}=\frac{\rho\left(19683 \rho^{2}+82377 \rho+115641\right)+54739-[1657+9 \rho(81 \rho+242)] \Psi}{2304 c}
$$

where $\Psi \equiv \sqrt{9 \rho(81 \rho+194)+1081}$. Resorting to numerical simulations, it can be ascertained that $\Pi_{1 L}>\pi_{H}$ in the admissible range of parameters. ${ }^{3}$ Equilibrium market shares in the duopoly phase are:

$$
x_{L}^{\mathfrak{l}}=\frac{\Psi-27 \rho-19}{24} ; x_{H}^{\mathfrak{f}}=\frac{27 \rho+43-\Psi}{24} ; x_{L}^{\mathfrak{l}}>x_{H}^{\mathfrak{f}} \text { always. }
$$

Finally, $\widehat{\theta} \in(\underline{\theta}, \bar{\theta})$ always.

\subsection{High-quality leadership}

Now $\pi_{1}^{D}=\pi_{H}$ and $\pi_{2}^{D}=\pi_{L}$. The leader's problem consists in: ${ }^{4}$

$$
\begin{aligned}
\max _{q_{H}} \Pi_{1 H} & =(\bar{\theta}-1) q_{H}-c q_{H}^{2}+\frac{\left(q_{H}-q_{L}\right)\left[\bar{\theta}+1-c\left(q_{H}+q_{L}\right)\right]^{2}}{9(1+\rho)} \\
\text { s.t. } & : \quad \frac{\partial \pi_{L}}{\partial q_{L}}=0 \Leftrightarrow q_{L}^{*}=\frac{\bar{\theta}-2+c q_{H}}{3 c}
\end{aligned}
$$

Adopting the same procedure as in the previous case, we can find the optimal qualities:

$$
\begin{aligned}
q_{L}^{\mathfrak{f}} & =\frac{16 \bar{\theta}+27 \rho+11-\sqrt{9 \rho(81 \rho+226)+1369}}{32 c} \\
q_{H}^{\mathfrak{l}} & =\frac{16 \bar{\theta}+81 \rho+97-3 \sqrt{9 \rho(81 \rho+226)+1369}}{32 c}
\end{aligned}
$$

with $\lim _{\rho \rightarrow \infty} q_{H}=(\bar{\theta}-1) /(2 c)$. Equilibrium profits are:

$$
\begin{aligned}
\Pi_{1 H}= & \left\{1152 \bar{\theta}(\bar{\theta}-2)(1+\rho)-\rho\left(19683 \rho^{2}+82377 \rho+111753\right)+\right. \\
& \left.-48547+\Psi^{3}\right\} /[4608 c(1+\rho)]
\end{aligned}
$$

\footnotetext{
${ }^{3}$ The ranges are $\rho \in[0, \infty)$, i.e., $\delta \in[0,1]$, and $\bar{\theta} \geq 7 / 2$. The latter condition ensures that full coverage obtains in duopoly. If so, then it also holds under monopoly, where $\bar{\theta} \geq 3$ would suffice (see Lambertini, 1996, 1997).

${ }^{4}$ There exists another solution to $\partial \pi_{L} / \partial q_{L}=0$, i.e., $q_{L}=\left(\bar{\theta}-2-c q_{H}\right) / c$. Again, this can be excluded on the basis of second order conditions.
} 


$$
\pi_{L}=\frac{\rho\left(19683 \rho^{2}+88209 \rho+130761\right)+64027-(9 \rho+13)(81 \rho+133) \Phi}{2304 c}
$$

where $\Phi \equiv \sqrt{9 \rho(81 \rho+226)+1369}$. Again, numerical simulations show that $\Pi_{1 H}>\pi_{L}$ in the admissible range of parameters. ${ }^{5}$ Equilibrium market shares at $t=1$ are:

$$
x_{L}^{\mathfrak{l}}=\frac{27 \rho+43-\Phi}{24} ; x_{H}^{\mathfrak{f}}=\frac{\Psi-27 \rho-19}{24} ; x_{L}^{\mathfrak{l}}<x_{H}^{\mathfrak{f}} \text { always. }
$$

Again, $\widehat{\theta} \in(\underline{\theta}, \bar{\theta})$ always.

\subsection{The subgame perfect equilibrium and welfare as- sessment}

In order to complete the characterisation of the subgame perfect equilibrium, consider the following inequalities:

$$
\Pi_{1 L}>\Pi_{1 H} \text { and } \pi_{H}>\pi_{L} \text { for all } \rho \in[0, \infty) \text {. }
$$

This holds for any admissible value of $\bar{\theta}$. Accordingly, we may state:

Proposition 1 The first entrant prefers to supply a low-quality good, while the second entrant prefers to supply a high-quality good. Therefore, the subgame perfect equilibrium is unique and involves $q_{1}=q_{L}^{\mathfrak{l}} ; q_{2}=q_{H}^{\mathfrak{f}}$.

Now we pass on to examine the welfare performance of the market in the two cases. In general, the definition of the discounted social welfare over the two periods is:

$$
S W \equiv \pi_{1}^{M}+\frac{\pi_{1}^{D}+\pi_{2}^{D}}{1+\rho}+C S
$$

\footnotetext{
${ }^{5}$ In this case, the result is intuitive, in that high-quality supply combines with a period of monopoly power, hence the first entrant's profits are necessarily higher than the second entrant's.
} 
where discounted consumer surplus is:

$C S \equiv \int_{\bar{\theta}-1}^{\bar{\theta}}\left(s q_{1}-p_{M}\right) d s+\frac{1}{1+\rho}\left(\int_{\bar{\theta}-1}^{\widehat{\theta}}\left(s q_{L}-p_{L}\right) d s+\int_{\widehat{\theta}}^{\bar{\theta}}\left(s q_{H}-p_{H}\right) d s\right)$

The relevant equilibrium expressions can be calculated using equilibrium prices and qualities in the two settings, to obtain:

Proposition 2 Discounted social welfare is higher when the leader chooses to offer the low-quality good than the high-quality one, for all admissible values of $\rho$ and $\bar{\theta}$.

Therefore, there is no conflict between private and social incentives as to the sequence of entry. The reason is that average quality is higher when the leader enters with a low quality, as it can be ascertained from the difference $\left(q_{L}^{\mathfrak{l}}+q_{H}^{\mathfrak{f}}\right) / 2-\left(q_{L}^{\mathfrak{f}}+q_{H}^{\mathfrak{l}}\right) / 2$, which is always positive in the admissible parameter range. 


\section{References}

[1] Cremer, H. and J.-F. Thisse, 1991, Location models of horizontal differentiation: a special case of vertical differentiation models, Journal of Industrial Economics, 39, 383-90.

[2] Donnenfeld, S. and S. Weber, 1992, Vertical product differentiation with entry, International Journal of Industrial Organization, 10, 449-72.

[3] Dutta, P.K., S. Lach and A. Rustichini, 1995, Better late than early: vertical differentiation in the adoption of a new technology, Journal of Economics and Management Strategy, 4, 563-589.

[4] Gabszewicz, J.J. and J.-F. Thisse, 1979, Price competition, quality and income disparities, Journal of Economic Theory, 20, 340-59.

[5] Gabszewicz, J.J. and J.-F. Thisse, 1980, Entry (and exit) in a differentiated industry, Journal of Economic Theory, 22, 327-38.

[6] Lambertini, L., 1996, Choosing roles in a duopoly for endogenously differentiated products, Australian Economic Papers, 35, 205-24.

[7] Lambertini, L., 1997, The multiproduct monopolist under vertical differentiation: an inductive approach, Recherches Economiques de Louvain, 63, 109-22.

[8] Shaked, A. and J. Sutton, 1982, Relaxing price competition through product differentiation, Review of Economic Studies, 49, 3-13.

[9] Shaked, A. and J. Sutton, 1983, Natural oligopolies, Econometrica, 51, 1469-83. 